\title{
Reconstruction techniques in supersymmetry searches in the ATLAS experiment
}

\author{
Eirik Gramstad ${ }^{* \dagger}$ \\ University of Oslo \\ E-mail: egramsta@cern.ch
}

\begin{abstract}
Many supersymmetric scenarios feature final states with non-standard final state objects. The production of massive sparticles can lead to the production of boosted top quarks or vector bosons and high-pt b-jets. At the same time, transitions between nearly mass-degenerate sparticles can challenge the standard reconstruction because of the presence of very soft leptons or jets. The talk will review the application of innovative reconstruction techniques to supersymmetry searches within the ATLAS experiment at the LHC.
\end{abstract}

XXVI International Workshop on Deep-Inelastic Scattering and Related Subjects (DIS2018) 16-20 April 2018

Kobe, Japan

\footnotetext{
*Speaker.

${ }^{\dagger}$ On behalf of the ATLAS Collaboration
} 


\section{Introduction}

Run 2 of the Large Hadron Collider (LHC) [1] at CERN is now in its last year of running before a 2-year shutdown. From 2015 to 2017 the LHC delivered $80 \mathrm{fb}^{-1}$ of data to be used for physics analyses. Despite all the data which has so far been collected by the ATLAS experiment [2] none of the numerous analyses which have been conducted show any significant evidence for new physics beyond the Standard Model (SM). With an expected doubling of the luminosity by the end of 2018 analyzers will get even more data, to be analyzed in great detail during the long shutdown. One of the theories which occupy a majority of physicists looking for physics beyond SM is Supersymmetry (SUSY). SUSY postulates a symmetry between fermions and bosons, predicting a new set of supersymmetric particles closely linked to their SM partners with an exception of spin, which differs by $1 / 2$. However, the so far negative results from all searches for supersymmetric partners suggest that the symmetry must be broken, allowing the SUSY particles to acquire a larger mass than their SM partners [3]. Despite decades of negative results from SUSY searches from many different experiments it is still one of the most vital theories beyond the SM, mostly because it provides a very attractive and elegant solution to the hierarchy problem [4-7] and predicts the existence of a Weakly Interacting Massive Particle (WIMP), a good candidate the dark matter in the universe $[8,9]$.

The absence of any evidence for SUSY has motivated physicists to not just wait for more and more data to be collected, but to develop better reconstruction techniques taking into account the fact that many supersymmetric scenarios feature final states with non-standard event kinematics. To obtain better sensitivity to larger parts of the SUSY phase space, events are being reconstructed by novel techniques taking advantage of recent developments within event reconstruction in ATLAS. In the following some of the applications of innovative reconstruction techniques to supersymmetry searches in ATLAS are presented.

\section{Boosted Objects}

The shower of particles originating from the hadronisation of a strongly-interacting particle produced in a high-energy particle collision is reconstructed experimentally as a jet. In the ATLAS detector this is performed using various recombination procedures, for which most SUSY analyses use the anti- $k_{t}$ algorithm. Common for all the recombination procedures is a predefined distance parameter, $R$, which determines the ultimate size in $(y, \phi)$-space of the output jets. However, the optimal $R$ is process-dependent and scales (for a two-body decay) as the inverse of the momentum under consideration. This means that ideally the $R$ parameter would be optimized for each and every analysis. This is not feasible because each jet configuration needs to be calibrated to account for detector response, pile-up suppression and other experimental effects. Most analyses in ATLAS use an $R$ parameter of 0.4. A method enabling use of different values of $R$ for their jets has been developed, where one builds large $R$-jets from smaller, calibrated, $R$-jets through re-clustering algorithms [10]. Using this method the calibration of the small $R$-jets automatically gives the corresponding calibration of the re-clustered large $R$-jet. Comparing $R=1.0$-jets built directly using topo-clusters from calorimeter cells with re-clustered $R=1.0$ jets reveals both smaller uncertainties on the jet mass and better resolution for the re-clustered jets [11]. Large $R$-jets are used in several 
analyses searching for SUSY in ATLAS. In searches for direct production of stop quarks [12] large $R$-jets $(R=3.0)$ are used to target events where top quarks are produced with a significant boost. The radius of each reconstructed large $R$-jet is iteratively reduced to an optimal radius matching their $p_{\mathrm{T}}$ given by $R\left(p_{\mathrm{T}}\right)=2 \frac{m_{t o p}}{p_{\mathrm{T}}}$. If a jet candidate looses a large fraction of its $p_{\mathrm{T}}$ in the reduction it is discarded. The same technique, but disregarding b-tagged jets, is used to identify boosted $W$ candidates. Using the re-clustered jets one can calculate the jet mass given in Eq. 2.1

$$
M_{J}=\left(\sum_{i \in j e t} E_{i}\right)^{2}-\left(\sum_{i \in j e t} \mathbf{p}_{i}\right)^{2}
$$

where $i$ runs over the constituents of the jet. Distribution of the mass of the reclustered top-quark, $m_{\text {top }}^{\text {reclustered }}$, candidate is shown in Figure 1a. Large $R$-jets and corresponding jet masses are also

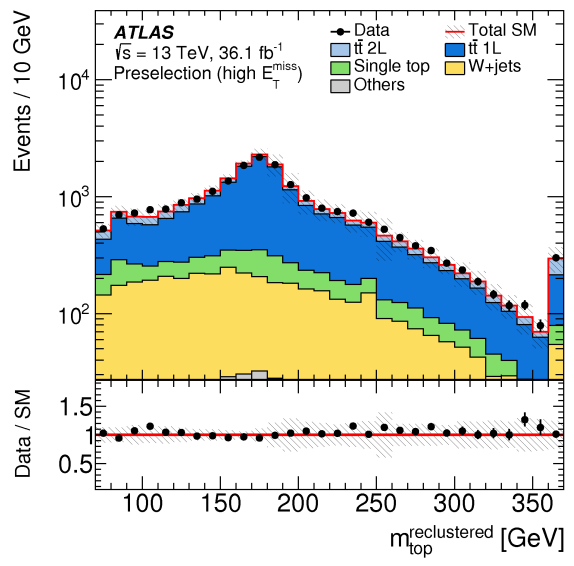

(a)

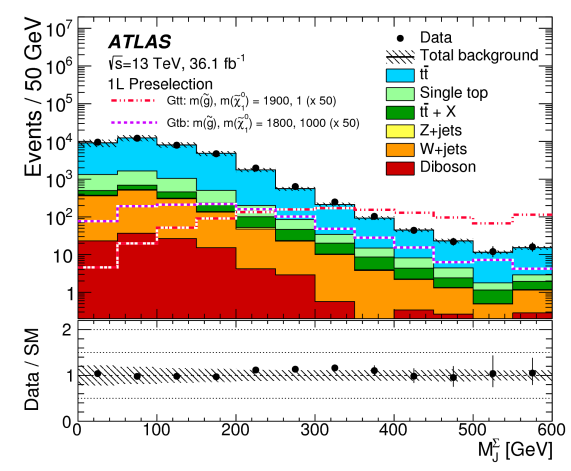

(b)

Figure 1: Plot in (a) shows the distribution of the mass of the reclustered top-quark candidate. [12] In (b) is the total jets mass for the SM backgrounds (colored histograms) and two signal models (dashed lines). [13]

used in analyses targeting models of direct gluino production where each gluino undergoes an effective three body decay, $\tilde{g} \rightarrow t \bar{t} \tilde{\chi}_{1}^{0}$, via off-shell top squarks with $100 \%$ branching fraction [13]. In these events the decay products of hadronically decaying boosted top quarks can be reconstructed within a single large $R$ re-clustered jet, resulting in a jet with high mass. This is then further used in constructing a total jet mass for the complete event, $M_{J}^{\Sigma}$, defined in Eq. 2.2

$$
M_{J}^{\sum}=\sum_{i \leq 4} m_{J, i}
$$

where $m_{J, i}$ is the mass of the $i^{\text {th }}$ re-clustered large $R$-jet in the event. Figure $1 \mathrm{~b}$ illustrates the good discriminating power of the total jet mass against $t \bar{t}$ for two signal models. Thanks to the re-clustering techniques several SUSY searches in ATLAS now use large $R$-jets to increase their sensitivity in many regions of phase space. These methods become particularly sensitive when the mass difference between the parent and child SUSY particle in the decay chain is large, i.e. where we expect the objects to be significantly boosted. 


\section{Recursive Jigsaw Reconstruction (RJR)}

The previous Section described SUSY searches targeting typical scenarios with large mass differences between the parent and the child SUSY particle. However, since we do not know the overall mass scale nor the mass hierarchy of the SUSY particles one should try to target also the more compressed regions, where the mass differences of the SUSY particles in the decay chain are small. Traditionally these scenarios have proven to be very challenging since one a priori expects little missing transverse energy $\left(E_{\mathrm{T}}^{\text {miss }}\right)$ and low momenta particles. To circumvent the problem of low momenta particles and small $E_{\mathrm{T}}^{\text {miss }}$ one in stead considers events with initial state radiation, where the high momentum of the SUSY decay chain comes from the recoil against a high momentum initial state radiation (ISR) jet. These scenarios typically motivate searches for monoISR-signals where one does not reconstruct the recoiling part of the event, but simply treat the $E_{\mathrm{T}}^{\text {miss }}$ recoiling against a high $p_{\mathrm{T}}$ jet. The Recursive Jigsaw Reconstruction (RJR) technique [1416], however, tries to separate the ISR objects from the sparticle objects. This is accomplished by considering each event to follow a decay tree as sketched in Figure 2a which first involves a transformation from the lab to the center of mass (CM) frame. The CM system includes all visible objects as well as the $E_{\mathrm{T}}^{\text {miss }}$. The objects are then further classified into two groups depending on whether or not they come from the SUSY decay chain (S) or not (ISR). The SUSY decay chain system is further divided into a visible (V) and an invisible (I) system, where the latter is assigned to the system of the LSPs produced in the event. Each event is then reconstructed by first ignoring the longitudinal momenta of all jets and treating $E_{\mathrm{T}}^{\text {miss }}$ as the transverse momentum of I (assuming zero mass). All jets are then further partitioned into ISR and $V$ by minimizing the mass of the ISR and S systems. Finally the event is analyzed in the transverse view and several kinematics variables are constructed. One such variable is the $p_{T S}^{C M}$ which is the magnitude of the vector sum of the $E_{\mathrm{T}}^{\mathrm{miss}}$ and the transverse momenta of all jets associated to the S system, evaluated in the CM frame. The good discriminating power of this variable is clearly illustrated in Figure $2 \mathrm{~b}$ using a

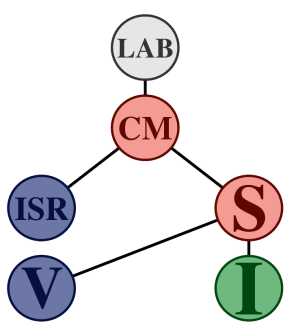

(a)

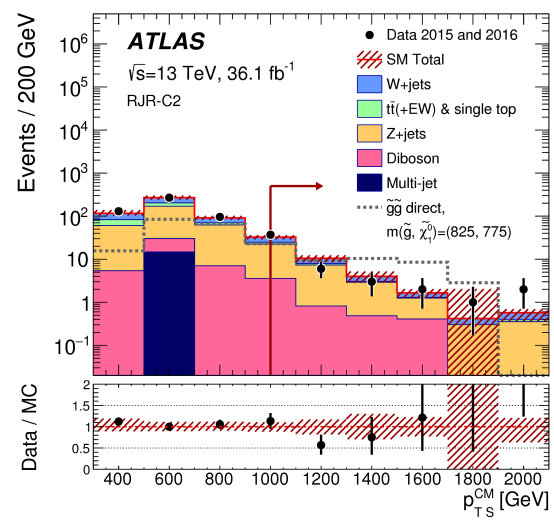

(b)

Figure 2: The sketch in (a) shows a simplified view of an event used to construct the RJR variables. In (b) is the distribution of $p_{T S}^{C M}$ for the SM backgrounds (colored histograms) and a SUSY signal model (dashed line). The red arrow indicates the cut used in the analysis. From [17].

signal model of direct production of gluinos decaying into quarks and the LSP with a mass splitting 
of only $50 \mathrm{GeV}$ between the gluino and the LSP.

The RJR techniques are used in several searches for SUSY in ATLAS with jets, $E_{\mathrm{T}}^{\text {miss }}$ and/or leptons in the final state $[12,17-19]$.

\section{Searches with b- and c-tagged jets}

In supersymmetric models, naturalness arguments suggest that the superpartners of the third generation quarks (stop $(\tilde{t})$ and sbottom $(\tilde{b})$ ) are the lightest colored supersymmetric particles [20, 21]. This would result in $\tilde{t}$ and $\tilde{b}$ quarks being produced at a relatively high rate at the LHC giving final states with several b- and/or c-jets. Since the start-up of the LHC the b- and c-tagging performance has increased significantly, especially with the installation of the insertable b-layer (IBL) [22]. Since then the use of new reconstruction techniques and by exploiting new approaches to multivariate analyses and training samples have further increased both the b- and c-jet tagging efficiencies as well as the rejection against lighter quarks and tau leptons. The increased performance in b- and c-tagging has resulted in increased sensitivity in analyses targeting direct production of charm, bottom or top squarks. The latter model is particular interesting in the cases were the mixing between the top and the charm squark is large. This would lead to cases were the two-body decay of a stop into a charm quark and the LSP is favored with respect to the three- and four-body decays, $\tilde{t} \rightarrow W b \tilde{\chi}_{1}^{0}$ and $\tilde{t} \rightarrow b f \bar{f}^{\prime} \tilde{\chi}_{1}^{0}$, respectively [23].

\section{Conclusions}

Although LHC has delivered large amounts of data at $\sqrt{s}=13 \mathrm{TeV}$ analyzers are constantly developing better and more sophisticated techniques to probe new SUSY parameter space. This article has discussed a few of these new techniques, which all have been used by one or several analyses searching for SUSY recently published by the ATLAS experiment. All these analyses have significantly extended the parameter space, in particular in cases where SUSY predicts very boosted objects, a compressed sparticle spectra or several high $p_{\mathrm{T}}$ b- or c-jets. With the upcoming long shutdown of the LHC the invention and development of novel reconstruction techniques will be even more important in order to fully utilize all the data delivered by the LHC.

\section{References}

[1] L. Evans and P. Bryant, LHC Machine, Journal of Instrumentation 3 (2008) S08001.

[2] ATLAS Collaboration, The ATLAS experiment at the CERN large hadron collider, Journal of Instrumentation 3 (2008) S08003.

[3] L. Girardello and M. T. Grisaru, Soft Breaking of Supersymmetry, Nucl. Phys. B 194 (1982) 65.

[4] N. Sakai, Naturalness in Supersymmetric Guts, Z. Phys. C 11 (1981) 153.

[5] S. Dimopoulos, S. Raby and F. Wilczek, Supersymmetry and the Scale of Unification, Phys. Rev. D 24 (1981) 1681.

[6] L. E. Ibanez and G. G. Ross, Low-Energy Predictions in Supersymmetric Grand Unified Theories, Phys. Lett. B 105 (1981) 439. 
[7] S. Dimopoulos and H. Georgi, Softly Broken Supersymmetry and SU(5), Nucl. Phys. B 193 (1981) 150.

[8] H. Goldberg, Constraint on the Photino Mass from Cosmology, Phys. Rev. Lett. 50 (1983) 1419.

[9] J. R. Ellis, J. S. Hagelin, D. V. Nanopoulos, K. A. Olive and M. Srednicki, Supersymmetric Relics from the Big Bang, Nucl. Phys. B 238 (1984) 453.

[10] B. Nachman, P. Nef, A. Schwartzman, M. Swiatlowski and C. Wanotayaroj, Jets from jets: re-clustering as a tool for large radius jet reconstruction and grooming at the lhc, Journal of High Energy Physics 2015 (2015) 75.

[11] ATLAS Collaboration, Jet reclustering and close-by effects in ATLAS run II, Tech. Rep. ATLAS-CONF-2017-062 (http://cds.cern.ch/record/2275649), CERN, Geneva, Jul, 2017.

[12] ATLAS Collaboration, Search for top-squark pair production in final states with one lepton, jets, and missing transverse momentum using $36 \mathrm{fb}^{-1}$ of $\sqrt{\mathrm{s}}=13 \mathrm{tev}$ pp collision data with the ATLAS detector, Journal of High Energy Physics 2018 (2018) 108.

[13] ATLAS Collaboration, Search for supersymmetry in final states with missing transverse momentum and multiple b-jets in proton-proton collisions at $\sqrt{s}=13$ tev with the atlas detector, Journal of High Energy Physics 2018 (2018) 107.

[14] M. R. Buckley, J. D. Lykken, C. Rogan and M. Spiropulu, Super-razor and searches for sleptons and charginos at the lhc, Phys. Rev. D 89 (2014) 055020.

[15] P. Jackson, C. Rogan and M. Santoni, Sparticles in motion: Analyzing compressed susy scenarios with a new method of event reconstruction, Phys. Rev. D 95 (2017) 035031.

[16] P. Jackson and C. Rogan, Recursive Jigsaw Reconstruction: HEP event analysis in the presence of kinematic and combinatoric ambiguities, Phys. Rev. D96 (2017) 112007.

[17] ATLAS Collaboration, Search for squarks and gluinos in final states with jets and missing transverse momentum using $36 \mathrm{fb}^{-1}$ of $\sqrt{\mathrm{s}}=13 \mathrm{TeV}$ pp collision data with the ATLAS detector, Physical Review D 97 (2018) .

[18] ATLAS Collaboration, Search for a scalar partner of the top quark in the jets plus missing transverse momentum final state at $\sqrt{s}=13$ tev with the ATLAS detector, Journal of High Energy Physics 2017 (2017) 85 .

[19] ATLAS Collaboration, Search for chargino-neutralino production using recursive jigsaw reconstruction in final states with two or three charged leptons in proton-proton collisions at $\sqrt{s}=13$ TeV with the ATLAS detector, CERN-EP-2018-113 (2018) [1806.02293].

[20] R. Barbieri and G. Giudice, Upper bounds on supersymmetric particle masses, Nuclear Physics B 306 (1988) 63 .

[21] B. de Carlos and J. Casas, One-loop analysis of the electroweak breaking in supersymmetric models and the fine-tuning problem, Physics Letters B 309 (1993) 320 .

[22] M. Capeans, G. Darbo, K. Einsweiller, M. Elsing, T. Flick, M. Garcia-Sciveres et al., ATLAS Insertable B-Layer Technical Design Report, Tech. Rep. CERN-LHCC-2010-013. ATLAS-TDR-19, Sep, 2010.

[23] ATLAS Collaboration, Search for supersymmetry in final states with charm jets and missing transverse momentum in $13 \mathrm{TeV}$ p p collisions with the ATLAS detector, CERN-EP-2018-034 (2018) [1805.01649]. 